\title{
The signal transducers Stat1 and Stat3 and their novel target Jmjd3 drive the expression of inflammatory genes in microglia
}

\author{
Piotr Przanowski • Michal Dabrowski - Aleksandra Ellert-Miklaszewska • Michal Kloss • \\ Jakub Mieczkowski • Beata Kaza • Anna Ronowicz • Feng Hu • Arkadiusz Piotrowski • \\ Helmut Kettenmann • Jan Komorowski • Bozena Kaminska
}

Received: 13 May 2013 /Revised: 30 August 2013 / Accepted: 19 September 2013 /Published online: 6 October 2013

(C) The Author(s) 2013. This article is published with open access at Springerlink.com

\begin{abstract}
Most neurological diseases are associated with chronic inflammation initiated by the activation of microglia, which produce cytotoxic and inflammatory factors. Signal transducers and activators of transcription (STATs) are potent regulators of gene expression but contribution of particular STAT to inflammatory gene expression and STAT-dependent transcriptional networks underlying brain inflammation need to be identified. In the present study, we investigated the genomic distribution of Stat binding sites and the role of Stats in the gene expression in
\end{abstract}

Electronic supplementary material The online version of this article (doi:10.1007/s00109-013-1090-5) contains supplementary material, which is available to authorized users.

P. Przanowski • M. Dabrowski • A. Ellert-Miklaszewska •

M. Kloss $\cdot$ J. Mieczkowski $\cdot$ B. Kaza

Laboratory of Molecular Neurobiology, Nencki Institute of

Experimental Biology, Warsaw, Poland

\section{A. Ronowicz $\cdot$ A. Piotrowski}

Faculty of Pharmacy, Medical University of Gdansk, Gdansk, Poland

F. $\mathrm{Hu} \cdot \mathrm{H}$. Kettenmann

Cellular Neurosciences, Max Delbrück Centre for Molecular

Medicine, Berlin, Germany

J. Komorowski

Department of Cell and Molecular Biology, Uppsala University,

Uppsala, Sweden

\section{J. Komorowski}

Interdisciplinary Centre for Mathematical and Computational

Modeling, Warsaw University, Warsaw, Poland

B. Kaminska $(\bowtie)$

Neurobiology Center, Nencki Institute of Experimental Biology,

Pasteur 3 str., Warsaw 02-093, Poland

e-mail: bozenakk@nencki.gov.pl

B. Kaminska

Laboratory of Molecular Neurobiology, Nencki Institute of

Experimental Biology, Warsaw, Poland lipopolysaccharide (LPS)-activated primary microglial cultures. Integration of chromatin immunoprecipitation-promoter microarray data and transcriptome data revealed novel Stat-target genes including Jmjd3, Ccl5, Ezr, Ifih1, Irf7, Uba 7, and Pim1. While knockdown of individual Stat had little effect on the expression of tested genes, knockdown of both Stat1 and Stat3 inhibited the expression of Jmjd 3 and inflammatory genes. Transcriptional regulation of Jmjd3 by Stat1 and Stat3 is a novel mechanism crucial for launching inflammatory responses in microglia. The effects of Jmjd3 on inflammatory gene expression were independent of its H3K27me3 demethylase activity. Forced expression of constitutively activated Stat1 and Stat 3 induced the expression of Jmjd3, inflammation-related genes, and the production of proinflammatory cytokines as potently as lipopolysacharide. Gene set enrichment and gene function analysis revealed categories linked to the inflammatory response in LPS and Stat1C + Stat3C groups. We defined upstream pathways that activate STATs in response to LPS and demonstrated contribution of Tlr4 and Il-6 and interferon- $\gamma$ signaling. Our findings define novel direct transcriptional targets of Stat1 and Stat3 and highlight their contribution to inflammatory gene expression.

\section{Key Message}

- Combined analysis of genomic Stat occupancy and transcriptome revealed novel Stat target genes in LPSinduced microglia.

- Jmjd3 transcription factor is a novel transcriptional target of Stat1 and Stat3.

- Stat1 and Stat3 cooperate with Jmjd3 to induce the expression of pro-inflammatory genes.

- Constitutively active Stat 1 and Stat 3 fully mimic the LPSinduced upregulation of inflammatory genes and secretion of cytokines.

Keywords Inflammation - Signal transducers and activators of transcription · ChIP-chip · Jmjd3 H3K27me3 histone demethylase $\cdot$ Brain macrophages 


\section{Introduction}

The initiation of inflammation requires global activation of gene expression, postranscriptional regulation, epigenetic modifications, and changes in chromatin structure [1, 2]. The transcription factors nuclear factor kappa B (NF-kB)/ Rel, interferon-regulatory factor (IRF), and signal transducer and activator of transcription (STAT) [3-5] are wellestablished regulators of inflammatory gene expression, but the specific contribution of various factors to the transcriptional circuits underlying inflammation is poorly defined. STATs are latent transcription factors that are phosphorylated by activated cytokine receptor associated Janus kinase (JAK), tyrosine kinase growth factor receptors, and nonreceptor tyrosine kinases. Nearly 40 cytokine receptors signal through STATs, and these signaling cascades can be either pro- or anti-inflammatory [6-8]. Activated Stats have been implicated in the regulation of cell growth, differentiation, cell survival, and cross-talk between cancer and immune cells. Knockout studies have demonstrated that Stat1 plays an important role in inflammation, growth arrest, and the promotion of apoptosis; conversely, Stat3 promotes cell-cycle progression and cellular transformation and prevents apoptosis [8-10]. On the other hand, a conditional deletion of Stat 3 in macrophages/ neutrophils or in endothelial cells leads to exaggerated inflammation and leukocyte infiltration in multiple organs associated with increased production of pro-inflammatory cytokines that suggests the suppressive role of Stat3 [11, 12]. Stat1 and Stat3 activated by NF-kB-dependent interleukin-6 (IL-6) expression or by interferon- $\gamma$ (IFN- $\gamma$ ) have been implicated in inflammation $[13,14]$. Stat5a and Stat5b proteins alone are weak activators of transcription, and they act predominantly by cooperating and interacting with other proteins [15].

However, hundreds of genes have been identified by knockout and biochemical studies as potential Stat3 targets; only a small fraction has been proven to be direct Stat3 targets in various cells. Recently, molecular insight into the biology of Stats was gained from a meta-analysis of 29 available ChIP-seq data sets covering genome-wide occupancy of STATs $1,3,4,5 \mathrm{~A}, 5 \mathrm{~B}$, and 6 in several cell types including macrophages, $\mathrm{B}$ and $\mathrm{T}$ lymphocytes, murine embryonic fibroblasts, embryonic cells, and liver cells [16]. The study revealed that the majority of the classical STATbinding sites were located near genes with cell-specific expression, and each cell type displays a unique STAT binding pattern.

Most neurological diseases are associated with inflammation initiated by the activation of microglia_-brain resident, myeloid cells responsible for immune surveillance. Activated microglia initiate neuroinflammation by producing cytotoxic and inflammatory factors such as the cytokines IL- $1 \beta$, tumor necrosis factor alpha (TNF- $\alpha$ ), and IL-6, thereby aggravating brain damage [17]. Gene expression profiling and biochemical studies performed on lipopolysaccharide (LPS)-stimulated microglia in vitro and in an animal model of stroke demonstrated gross similarity in the transcriptional activation of a large panel of inflammatory genes [18]. It confirms that events underlying the inflammatory response in LPS-stimulated microglia reflect those that contribute to brain inflammation. The analysis of predominant signaling pathways revealed that Stat signaling and a subset of potentially Stat-dependent genes are activated in inflammatory microglia in vitro and in the ischemic brain.

To determine the Stat-dependent transcriptional network and its functions in inflammation, we investigated the genomic distribution of the Stat1, Stat3, and Stat 5 binding sites and the role of Stats in the control of the gene expression in LPSstimulated primary microglial cultures. Combined analysis of gene expression profiles and the genomic Stat occupancy data led to the identification novel, direct Stat target genes. In particular, we found that Jmjd3, an H3K27me3 demethylase and transcription factor, is a novel transcriptional target of Stat1 and Stat3, and cooperates with Stats to induce the expression of pro-inflammatory genes. Moreover, our data demonstrate that constitutively active Stat1 and Stat3 fully mimic the LPS-induced upregulation of inflammatory genes and secretion of cytokines.

\section{Materials and methods}

\section{Cell culture and treatments}

Primary cultures of rat microglia were prepared from 1-day-old Wistar rat pups as described previously. Briefly, cells were isolated from cerebral cortices and plated in culture medium [Dulbecco's modified Eagle's medium (DMEM) with Glutamax and high-glucose formula $4.5 \mathrm{~g} / \mathrm{L}$, Gibco] supplemented with $10 \%$ fetal bovine serum (FBS) and antibiotics in poly-L-lysine-coated culture flasks. After 9-10 days, the loosely adherent microglial cells were recovered from confluent glial cultures by mild shaking and centrifugation. The microglial cells were suspended and plated at a density of $2-3 \times 10^{5}$ cells $/ \mathrm{cm}^{2}$ in 24-well plates or $60 \mathrm{~mm}$ dishes. Nonadherent cells were removed after $30 \mathrm{~min}$ by changing the medium. Adherent cells ( $>96 \%$ positive for isolectin $\mathrm{B}_{4}$ ) were incubated for $48 \mathrm{~h}$ to silence the microglial culture. Primary microglial cultures were prepared from cerebral cortex of newborn $\mathrm{C} 57 \mathrm{BL} / 6$ or TLR4 knockout mice. The TLR KO mice were generated on a C57Bl/6 background by Dr. Shizuo Akira and colleagues from the Osaka University, Japan and obtained from Oriental BioServices Inc., Japan [19]. Cells were stimulated with $100 \mathrm{ng} / \mathrm{ml}$ lipopolysaccharide (LPS from Salmonella enteritidis, Sigma, Germany). In some experiments, $10 \mu \mathrm{g} / \mathrm{ml}$ cycloheximide (CHX) (Sigma-Aldrich, St. Louis, MO, USA) 
was added together with LPS. Histone methyltransferase inhibitors 3-deazaneplanocin and BIX 01294 (Sigma, Germany) were added for $6 \mathrm{~h}$ at a final concentration $3 \mu \mathrm{M}$. Primary mouse microglia cultures were treated by murine recombinant IL-6 $(25 \mathrm{ng} / \mathrm{ml})$ and IFN- $\gamma(10 \mathrm{ng} / \mathrm{ml})$ for $1 \mathrm{~h}$ (cytokines were kindly provided by ImmunoTools, Germany). Murine BV2 immortalized microglial cells (obtained from Dr. Klaus Reymann) were cultured in DMEM supplemented with $2 \%$ FBS, $50 \mathrm{U} / \mathrm{ml}$ penicillin, and $50 \mathrm{mg} / \mathrm{ml}$ streptomycin. The cells were grown in 12-well plates (for RNA isolation) or 6-well plates (for protein extraction) at a density of $1 \times 10^{5}$ or $2.5 \times 10^{5}$ cells per well, respectively, in a humidified atmosphere containing $5 \% \mathrm{CO}_{2}$.

Preparation of protein extracts and Western blot analysis

Cell lysates were prepared by scraping the cells in buffer containing phosphatase and protease inhibitors. The proteins were then separated by sodium dodecyl sulfate polyacrylamide gel electrophoresis and transferred onto nitrocellulose membranes as described previously [20]. Antibodies used for Western blot analysis were purchased from Cell Signaling Technology (Beverly, MA, USA) and included the following: polyclonal antibodies recognizing phosphorylated and total Stat1, Stat3, Stat5, Jmjd3, phosphorylated IkB, and horseradish peroxidase-conjugated antirabbit IgG. Protein molecular weights were estimated with prestained protein markers. The membranes were stripped and reprobed with horseradish peroxidase-conjugated anti- $\beta$-Actin antibody (SigmaAldrich, St. Louis, MO, USA) to verify that equal amounts of protein were loaded.

Chromatin immunoprecipitation and hybridization to microarrays (ChIP-chip)

For ChIP-chip experiments, $1 \times 10^{7}$ cells were stimulated for $1.5 \mathrm{~h}$ with LPS $(100 \mathrm{ng} / \mathrm{ml})$. Fixation with $1 \%$ formaldehyde, sonication, and immunoprecipitation were performed with components of the ChIP IT kit according to the manufacturer's instructions (Active Motif, Carlsbad, CA, USA). Each sample was immunoprecipitated with $1 \mu \mathrm{g}$ of one of the following antibodies: anti-P-Stat1 (sc-7988X), anti-PStat3 (sc-7993X), or anti-P-Stat5 (sc-11761X) from Santa Cruz Biotechnology (Santa Cruz, CA, USA). Normal rabbit IgG (NI01) from Calbiochem (Darmstadt, Germany) served as the control immunoprecipitation (IP) antibody. The amount and quality of the DNA were determined by capillary electrophoresis with a Bioanalyzer 2100 and a High Sensitivity DNA LabChip kit from Agilent Technologies (Santa Clara, CA, USA). For hybridization to microarrays, the material was amplified with a whole-genome amplification WGA3 kit (Sigma-Aldrich, St. Louis, MO, USA) according to the manufacturer's protocol. The sequences of the primers used for ChIP-PCR are presented in the supplementary Table 1.

The genomic positions of the Stat binding sites were identified by labeling and hybridizing the immunoprecipitated chromatin to Rat ChIP $3 \times 720 \mathrm{~K}$ RefSeq promoter oligonucleotide chips (Roche NimbleGen, Waldkraiburg, Germany); two-color competitive hybridization was performed with a labeled input as a reference. The chromosomal locations of the binding peaks for P-Stat1, P-Stat3, and P-Stat5 were identified with the program NimbleScan (version 2.5) using the default parameters. The chromosomal locations of the peaks were imported into a relational database and mapped to the nearest gene with Ensembl (version 60) after providing the software with the Nimblegen range $(-5,000$ to 1,000$)$ from the transcription start site of the gene. Integration of the ChIPseq data with gene expression data (Kolmogorov-Smirnov test) and data visualization were performed in Mathematica (Wolfram Research).

Transfection with plasmids and siRNA

Microglial BV2 cells were transfected with the Amaxa T kit (Lonza, Cologne, Germany) and the A-023 program according to the manufacturer's instructions. For overexpression experiments, cells were transfected with $5 \mu \mathrm{g}$ of DNA per $1 \times 10^{6}$ cells. The following plasmids were used: pEGFP-N1, Stat1C (kindly provided by Dr. Toru Ouchi, New York University, New York), Stat3C (kindly provided by Dr. James Darnell, The Rockefeller University, New York), Jmjd3, Jmjd3mut (deletion) (kindly provided by Dr. Susana Sola, University of Lisbon, Lisbon), and Jmjd3mut (catalytic) (kindly provided by Dr. Amy S. Weinmann, University of Washington, Seattle). The cells were collected for protein extracts and RNA isolation 18 and $24 \mathrm{~h}$ after transfection, respectively.

To silence individual Stats or Jmjd3, the cells BV2 were transfected by electroporation with $150 \mathrm{pmol}$ of a control smallinterfering RNA (siRNA) (SI03650325) or Stat 1 (SI00183547), Stat3 (SI01435287), and Jmjd3 (SI01079631) specific siRNAs purchased from Qiagen (Germantown, MD, USA). Transfected cells were seeded onto plates. After $48 \mathrm{~h}$, the transfected cells were stimulated with $100 \mathrm{ng} / \mathrm{ml}$ LPS for 3 or $6 \mathrm{~h}$ for protein extracts and $9 \mathrm{~h}$ for RNA collection.

RNA isolation, reverse transcription, and qPCR analysis

RNA was isolated and complementary DNA (cDNA) was obtained with a Ambion Cells-to-CT kit (Life Technologies, Carlsbad, CA, USA). Real-time PCR amplifications were performed in duplicate with cDNA as the template in a reaction volume of $10 \mu \mathrm{l}$; the reaction contained $2 \mathrm{x}$ SYBR GREEN FAST PCR Master Mix (Life Technologies, Carlsbad, CA, USA) and primer sets for the following genes: 
$18 S$ rRNA, iNOS, Irf7, Uba7, Jmjd3, Ccl5, Il-6, and Pim1 . The primer sequences are given in the Supplementary Table 1. The expression of all PCR products was normalized to $18 \mathrm{~S}$ rRNA and then to untreated controls. Fold changes were calculated by the $2^{-\Delta \Delta \mathrm{Ct}}$ method with 7500 System SDS software (Life Technologies). Statistical analyses were performed with Student's $t$ test with Statistica software (StatSoft, Tulsa, OK, USA). The results are expressed as the mean \pm standard deviation (SD). All experiments were performed with three independently derived microglial cultures or BV2 passages.

Microarray gene expression profiling in BV2 microglial cells

BV2 cells were transfected with Stat $1 \mathrm{C}$ and Stat $3 \mathrm{C}$ constructs or pEGFP-N1 (as a control); $24 \mathrm{~h}$ following transfection, the cells were stimulated with LPS for an additional $9 \mathrm{~h}$ or left untreated. Total RNA was isolated using RNeasy Mini Kit (Qiagen). The amount and quality of the RNA were determined by capillary electrophoresis with a Bioanalyzer 2100 and a RNA 6000 LabChip kit from Agilent Technologies. The microarray experiments were performed with $100 \mathrm{ng}$ of total RNA as the template. The whole-genome amplification procedure was performed with a GeneAtlas WT Expression Kit according to the manufacturer's User Guide for the GeneAtlas Personal Microarray System (Affymetrix, Santa Clara, CA, USA). Fragmented and labeled cDNA was hybridized to the Affymetrix Mouse Gene 1.1 ST Array Strip (770,317 probes including 28,853 mouse genes). Microarray data were analyzed according to the previously described RMA preprocessing method [21]. Briefly, probe set measurements were transformed into gene-specific measurements using the annotation provided in the Ensembl database. To remove potential crosshybridization effects, all probe sets with annotations for more than one gene were excluded from further analyses. Multiple probe sets were annotated for the same gene; therefore, we choose the best probe set, which was defined as the probe set with the smallest $p$ value obtained from the Welch's $t$ test, from each of these sets to obtain genespecific measurements. Changes in gene expression were evaluated with the limma package in the Bioconductor software [22]. We then computed $q$ values for all of the analyzed genes. The gene set enrichment analysis (GSEA, described by [23]) and overrepresentation analysis were performed with the MSigDB gene collections (http://www. broad.mit.edu/gsea/msigdb/index.jsp). The preprocessing methods and all of the statistical computations (except GSEA) were performed within the R programming environment and Bioconductor packages. GSEA was performed with the Java code provided by its authors. Microarray data have been deposited under the accession number E-MEXP-3659 (The ArrayExpress Archive, Hixton, UK).
Global H3K27me3 profiling by flow cytometry

To determine global H3K27me3 changes, cells were collected after stimulation for $9 \mathrm{~h}$ with LPS or $24 \mathrm{~h}$ post-plasmid transfection and fixed in $2 \%$ formaldehyde in phosphatebuffered saline (PBS) for $10 \mathrm{~min}$ at room temperature. To stop the reaction, $125 \mathrm{mM}$ glycine was added, and ice-cold $100 \%$ ethanol was slowly added to cells at a ratio of 1:1 (ethanol/ glycine). Cells were resuspended in MACS buffer (PBS, $125 \mathrm{mM}$ EDTA, and $0.5 \%$ bovine serum albumin), aliquoted among two tubes (with or without primary antibody, which served as a negative control), and permeabilized in MACS buffer containing $0.1 \%$ Triton X-100. The cells were incubated with anti-trimethyl histone $\mathrm{H} 3$ (Lys 27) primary antibody [Upstate (Millipore), Billerica, MA, USA] diluted 1:400 in MACS buffer for $1 \mathrm{~h}$ at $4{ }^{\circ} \mathrm{C}$ and rinsed four times with MACS buffer. Cells were incubated at room temperature for $1 \mathrm{~h}$ with Alexa Fluor 647-conjugated secondary antibody (diluted 1:2,000, Invitrogen), washed four times in MACS buffer, and resuspended in $2 \%$ formaldehyde in PBS. Fluorescence was measured with BD FACSCalibur and CellQuest software.

\section{Multiplex cytokine flow cytometry assay}

Cytokine measurements were performed in conditioned media using the Procarta Immunoassay Kit (Affymetrix, Santa Clara, CA, USA) on a Luminex 100 flow cytometry analyzer (Madison, WI, USA) according to the manufacturer's instructions. Conditioned media were collected $24 \mathrm{~h}$ after the transfection of BV2 cells with specific plasmids or $24 \mathrm{~h}$ after treatment with LPS. The following pro-inflammatory cytokines were measured: IFN- $\gamma$, Il-10/CSIF, Il-1 $\beta / I L I F 2, ~ I l-6$, RANTES/Cc15, and TNF- $\alpha$. Statistical analyses were performed with Student's $t$ test with Statistica software (StatSoft, Tulsa, USA).

\section{Results}

Whole-genome identification of P-Stat1, P-Stat3, and P-Stat5 binding sites upon inflammatory stimulation of rat primary microglial cultures

Western blot analysis demonstrated a rapid increase in the levels of phosphorylated Stat1, Stat3, and Stat5 from 1 to $6 \mathrm{~h}$, with the maximum induction occurring between 1 and $1.5 \mathrm{~h}$ after stimulation of rat primary microglial cultures with LPS (Fig. 1a). The direct Stat targets and their role in the transcriptional responses of microglial cells are unknown. Therefore, we analyzed the distribution of P-Stat1, P-Stat3, and P-Stat5 binding sites in promoter regions by chromatin immunoprecipitation followed by hybridization to promoter arrays (ChIP-chip analysis). Microglial cells 
were stimulated with LPS for $1.5 \mathrm{~h}$, when the peak of Stat phosphorylation was observed (Fig. 1a). Using a false discovery ratio (FDR) of $\leq 0.2$, we mapped to the genome 3,527 peaks (corresponding to 3,466 genes) for P-Stat1, 1,074 peaks (corresponding to 1,166 genes) for P-Stat3, and 939 peaks (corresponding to 1,012 genes) for P-Stat5 chromatin immunoprecipitation (Fig. 1b).

To identify genes whose transcription is directly regulated by distinct Stats, we analyzed the current ChIP-chip data together with global gene expression data from LPSstimulated primary rat microglial cultures. First, we evaluated whether the profile of changes in gene expression following LPS stimulation (microarray data E-MEXP-2466 from [18]) was significantly different in the sets of genes with and without the P-Stat1, P-Stat3, and P-Stat5 binding peaks. Using the Kolmogorov-Smirnov test, we compared the distributions of the log2-transformed fold changes in the expression of the gene sets with or without P-Stat binding peaks (Fig. 1c). The comparison was performed either (1) for all of the genes represented both in the ChIPchip and expression dataset or (2) following an optional filtration step that included only the genes with significantly altered expression. When analyzed for all genes, the two distributions were not significantly different. However, among the genes that exhibited significantly altered expression ( $t$ test $p<0.0001$, FDR $<0.0003$ ) following LPS treatment, the distributions of the log2-transformed fold changes in gene expression were clearly different between the genes with and without P-Stat1 and P-Stat3 binding peaks. We did not observe this relationship for P-Stat5 binding peaks (Fig. 1c).

To further explore the effect of P-Stat binding on gene expression for the set of the genes with significantly changed expression, we converted the $\log 2$ changes to ranks and used histograms to visualize the distribution of the ranks of the P-Stat binding genes among all the genes (Fig. 1c, lower panel). For random binding data (i.e., no association between the P-Stat binding and the expression rank), a uniform distribution of ranks (red horizontal lines in Fig. 1c) of the P-Stat binding genes would be expected. The genes with P-Stat1 and P-Stat3 binding sites were overrepresented among the genes most strongly upregulated by LPS treatment (in the top bins of the respective histograms). For both P-Stat1 and P-Stat3, the overrepresentation of the P-Stat binding genes in the top histogram bins was approximately twofold.

Figure 2 shows a binding map of P-Stat 1 and/or P-Stat 3 to the promoters of the 56 genes that exhibit P-Stat binding and are most strongly $(\mathrm{FC}>2)$ induced by LPS treatment in microglial cells. Heatmap representation shows the expression of 56 genes that exhibit P-Stat binding, and are most strongly $(\mathrm{FC}>2)$ induced by LPS in microglial cells (Fig. 2a). Despite the high similarity of the P-Stat1 and P-Stat 3 binding motifs, a majority of the genes with P-Stat binding peaks in their regulatory regions were found to bind either P-Stat1 or PStat3 but not both. A smaller subset of genes including Mmp13 and Jmjd3 was found to bind both P-Stat1 and PStat3.

Nine representative candidate genes, based on their known functions in inflammation, were selected for validation by PCR with independent biological samples. This group included Jmjd3 (two independent binding sites), Ccl5, Ezr, Ifih1, Irf7, Uba7, Il-6, and Pim1. The ChIPPCR confirmed the specific binding of Stat1 and/or Stat3 for eight of the nine promoters analyzed (Fig. 2b); the exception was Tlr7. In particular, we confirmed that PStat1 and P-Stat3 bind to the Jmjd3 gene promoter; one of the sites originally identified as a P-Stat3 binding site actually binds both P-Stat1 and P-Stat3. These results demonstrate a subset of genes upregulated in inflammatory microglial cells, which includes known (Il-6) and novel, transcriptional targets of Stats (Jmjd3, Ccl5, Ezr, Ifih1, Irf7, Uba7, and Pim1). These data suggest that Stat1 and/or Stat3 are required to induce expression of the target genes coding for inflammatory proteins, which are important in inflammation.

Silencing of Stat1, Stat3, or Jmjd3 affects the expression of selected targeted genes in LPS-induced microglial cells

Jmjd3, a JmjC family histone demethylase and a transcription factor, is induced by the transcription factor $\mathrm{NF}-\mathrm{kB}$ in response to microbial stimuli and has been shown to remove the histone $\mathrm{H} 3$ lysine 27 trimethylation (H3K27me3) that is associated with transcriptional repression in lineage determination [24]. More than $70 \%$ of the LPS-inducible genes were identified as Jmjd3 targets [25].

To determine the functional impact of the absence of Stat1, Stat3, and Jmjd3 on the transcriptional changes triggered by LPS stimulation, the expression of these three genes was silenced with specific siRNAs. Due to the poor transfectability of primary microglial cultures, genetic manipulation experiments were performed in murine immortalized BV2 microglial cells. These cells are frequently used for mechanistic studies of microglial biology and respond to LPS challenge in a manner similar to that of primary microglial cultures. We confirmed that LPS stimulation results in a rapid increase in the levels of P-Stat1, P-Stat3, and Jmjd3 in these cells, with kinetics similar to those observed in primary microglial cultures (Fig. 3a). The regulation of Jmjd3 by NF-kB was previously demonstrated in LPS-stimulated bone marrowderived macrophages [25]. We found that Jmjd3 is regulated by $\mathrm{NF}-\mathrm{\kappa B}$ in LPS-stimulated microglial cells, and Bay 11-7082 (an inhibitor of NF- $\mathrm{BB}$ activation) completely inhibited the LPS-induced Jmjd3 expression in BV2 cells (Fig. 3b). 
a

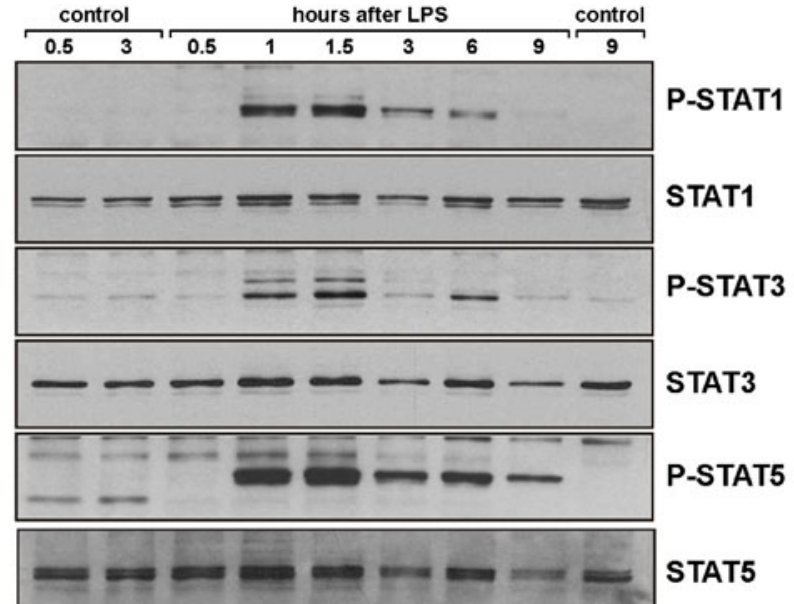

$-\infty-\ldots-\ldots-\beta$-actin

b

All genes with P-Stat peaks
in promoter regions

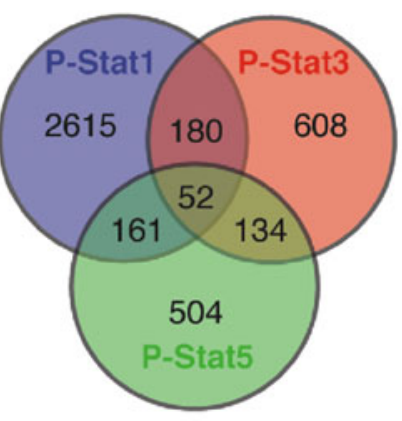

Genes with P-Stat peaks and reliable expression data

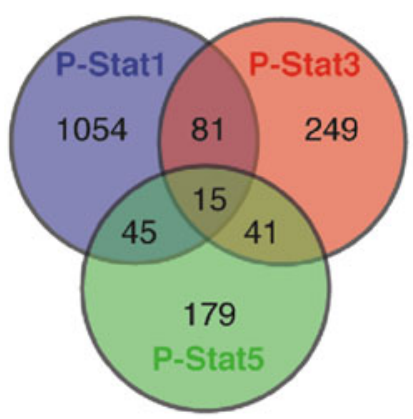

C
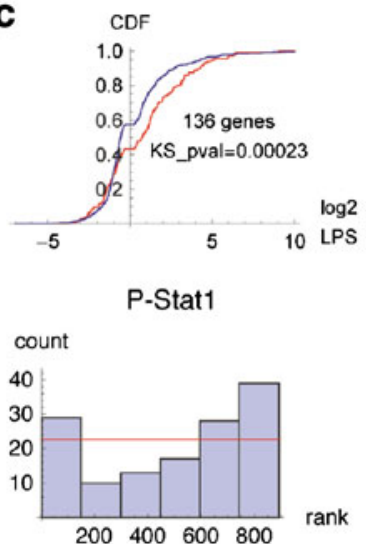

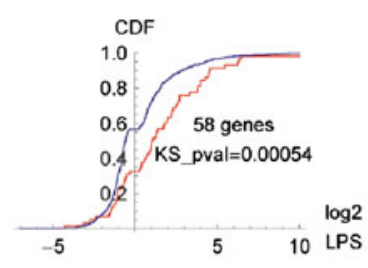

P-Stat3

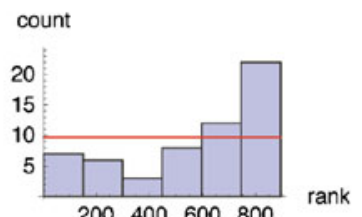

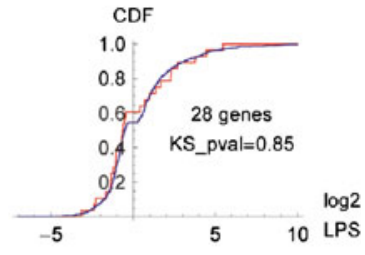

P-Stat5

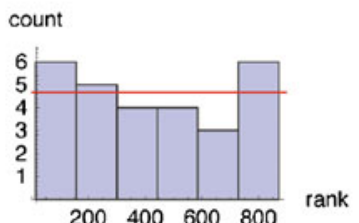

Silencing of Stat1 or Stat3 expression alone produced a small effect on Jmjd3 expression, but silencing both simultaneously profoundly reduced the level of Jmjd3 expression. This reduction in Jmjd3 expression was similar to the reduction observed following addition of Jmjd3-specific siRNA (Fig. 3c).
Furthermore, we investigated the effects of Stat1, Stat3, or Jmjd3 silencing on the expression of selected genes following LPS treatment: Il-6, iNos, Ccl5, Pim1, Uba7, and Irf7. Quantification of data from multiple independent experiments demonstrated that silencing of Stat1 or Stat3 alone had a weak 
Fig. 1 Integration of ChIP-chip data for P-Stat1, P-Stat3, and P-Stat5 with transcriptome analysis allows identification of novel Stat target genes in LPS-stimulated microglia. a Activation of Stat1, Stat3, and Stat5 in LPS-stimulated primary rat microglial cultures. The immunoblot shows the levels of phosphorylated and total Stats at various times after stimulation with $100 \mathrm{ng} / \mathrm{ml}$ LPS; membranes were reprobed with antiactin antibody to ensure equal loading of proteins. b Genome-wide occupancy of the Stat proteins and transcriptome analyses in primary rat microglial cultures. Chromatin immunoprecipitation and promoter arrays were used to identify the sequences bound by active Stat1, Stat 3 and Stat5 proteins in LPS-stimulated microglia. A diagram indicates the number of the genes bound by a given Stat and overlap between these genes (left panel). The ChIP-chip data for Stat binding were analyzed with the expression data from primary rat microglial cultures stimulated for $6 \mathrm{~h}$ with LPS $(100 \mathrm{ng} / \mathrm{ml})$ [18]. A diagram indicates the number of the genes with P-Stat peaks and reliable expression data (right panel). c Statresponsive genes (represented in the ChIP-chip experiment) significantly up- or downregulated in LPS-stimulated cells compared to control microglia (control vs. LPS $t$ test, $p<0.0001$ ). The cumulative distribution functions of the log2-transformed changes in the expression of the genes with (red) and without (blue) binding peaks for the given P-Stat, the numbers of genes containing peaks, and the $p$ values were calculated from the Kolmogorov-Smirnoff tests. Lower panel P-Stat binding peaks among the general set of genes $(t$ test $p<0.0001, \mathrm{FDR}<0.001)$ ranked in the increasing order on the LPS-induced $\log 2$ change in expression. Red horizontal lines show the uniform distributions that would be expected for random binding data effect on the expression of the selected genes, with the exception of the reduced expression of Il-6 and iNos in Stat3-depleted cells. Silencing of Stat1 and Stat 3 prevented the LPS-induced upregulation of the messenger RNA (mRNA) levels of the selected genes. Silencing of Jmjd3 yielded a moderate effect on the expression of these genes; this silencing effect that was smaller than the effect of combined silencing of both Stat1 and Stat3 (Fig. 3d).

Forced expression of constitutively active Stat1 and Stat3 induces Jmjd3 and inflammatory gene expression in BV2 microglial cells

To further investigate the role of Stat1, Stat3, and Jmjd3 in the response of microglial cells to LPS treatment, we used constructs encoding constitutively active forms of Stat1, Stat1C [26]; constitutively active Stat3, -Stat3C [10] and a wild-type Jmjd3 [27]. A pEGFP-N1 construct served as the control. In the absence of other stimuli, forced expression of constitutively active Stat1 and Stat3 induced Jmjd3 expression in BV2 microglial cells to a level similar to that observed following LPS treatment. Under these conditions, the NF-kB pathway was
Fig. 2 Verification of P-Stat1 and $\mathrm{P}-\mathrm{Stat} 3$ binding to selected gene promoters using ChIP-PCR. a Heatmap representation of the selected gene expression in nonstimulated cells (M, $n=6$ ) or LPSstimulated microglial cultures (L, $n=4)$. The correlation of the PStat1 and P-Stat 3 binding map for the genes with P-Stat1 or P-Stat3 binding sites that were most significantly $(t$ test $p<0.0001)$ and at least twofold induced by LPS. The map indicates the presence (black) or absence (white) of at least one P-Stat binding peak. b

Immunoprecipitated DNA samples were isolated from independently derived microglial cultures stimulated by LPS for 90 min. Immunoprecipitation with a neutral IgG served as a negative control ( $\mathrm{Neg}$ ); the input DNA was used as a positive control. The PCR products were resolved on $2 \%$ agarose with ethidium bromide. Similar results were obtained fromthree independent microglial cultures a

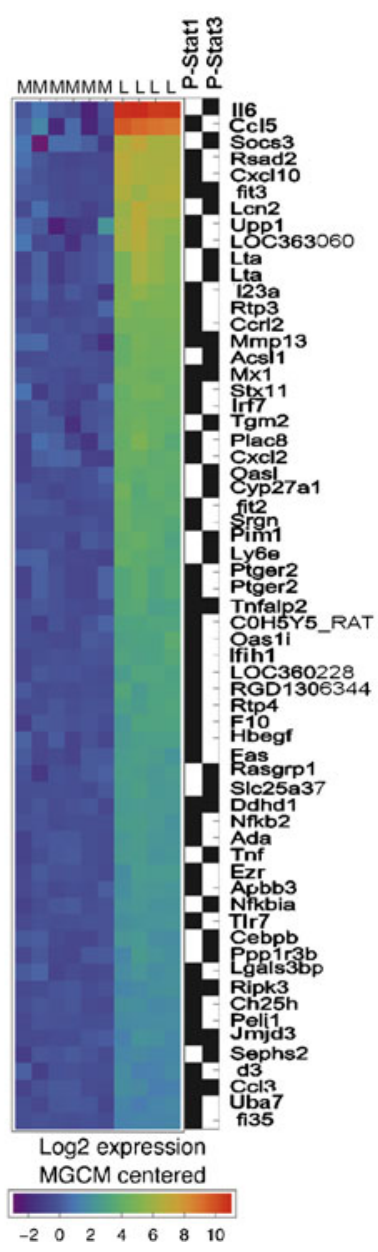

b

\section{Input Neg P-Stat1 P-Stat3}

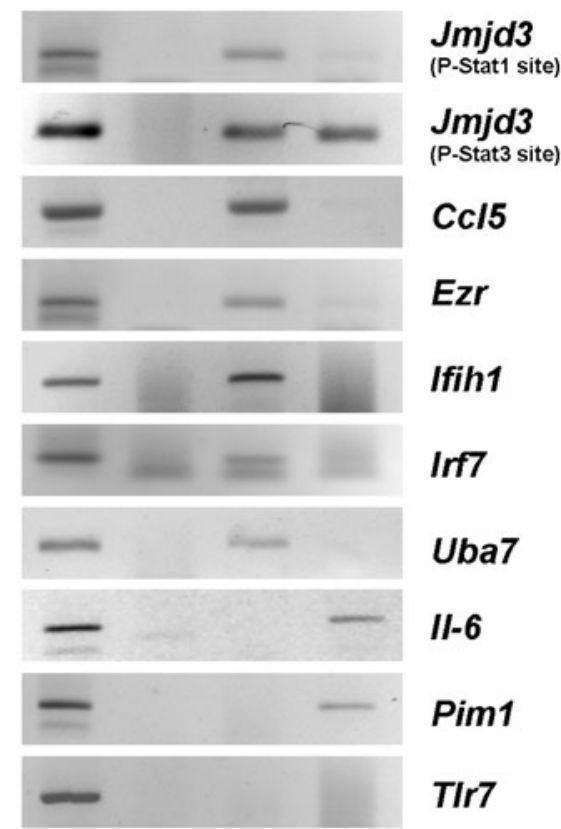




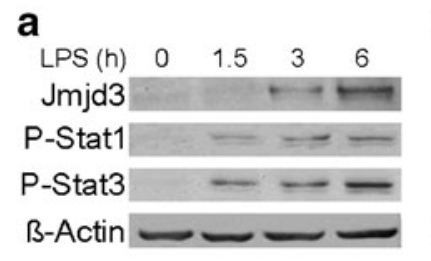

b

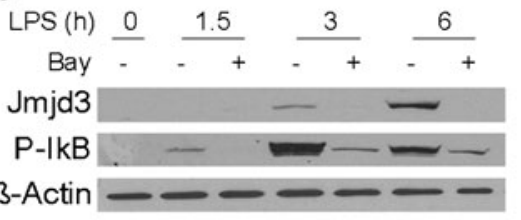

d

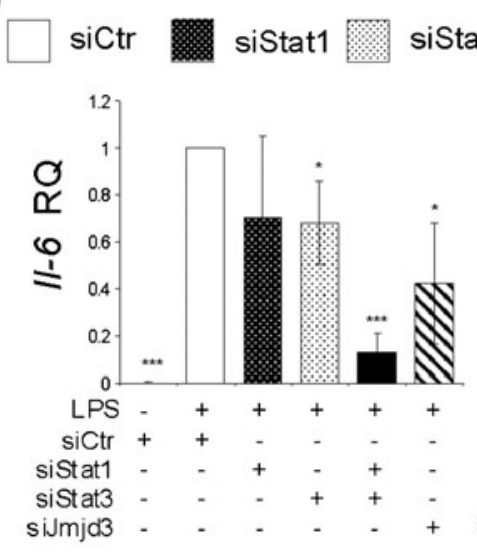

siJmjd3

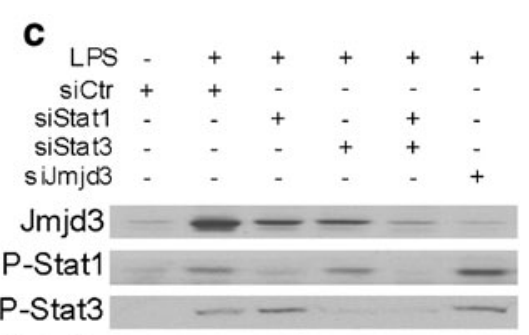

ß-Actin $=20$
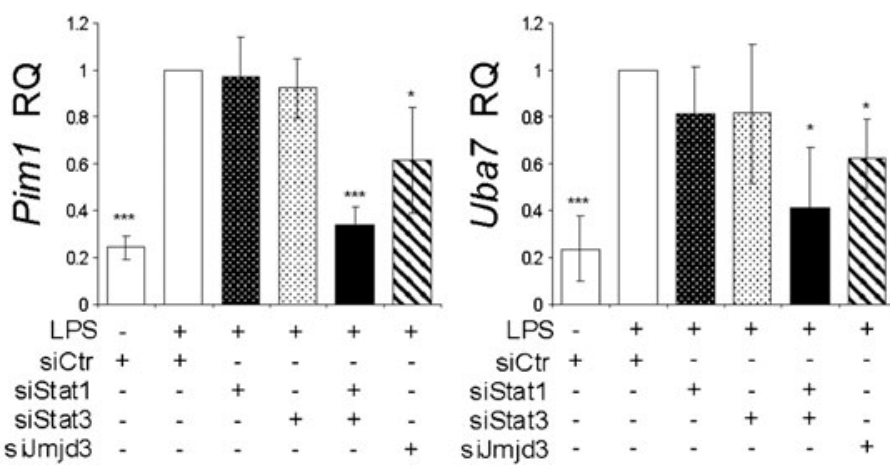
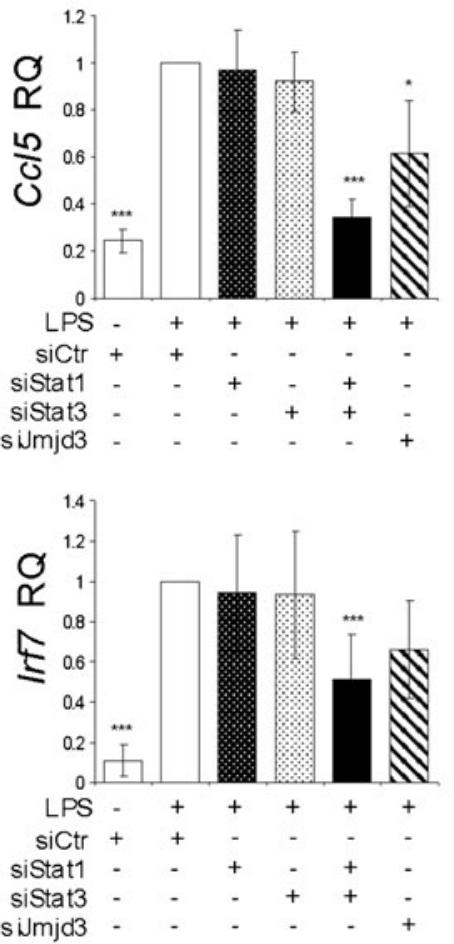

Fig. 3 Stat 1 and Stat3 regulate the expression of Jmjd3 and inflammation-related genes in LPS-stimulated BV2 microglial cells. a The levels of phosphorylated Stat1, Stat3, and Jmjd3 in total protein extracts from control and LPS-stimulated microglial BV-2 cells. b Bay 11-7082 (an inhibitor of NF-kB) blocks Jmjd3 expression. The immunoblot shows the reduced level of phosphorylated IKB and the absence of Jmjd 3 expression in LPS-stimulated microglial cells pretreated with Bay 11-7082. c Silencing of both Stat 1 and Stat3 expression prevents LPS-induced Jmjd3 expression as efficiently as pretreatment with siRNA against Jmjd3. Microglial cells were transfected by an Amaxa electroporation with control siRNA or siRNAs specific for Stat1, Stat3, or Jmjd3. After $48 \mathrm{~h}$, transfected cells were stimulated with LPS for $6 \mathrm{~h}$. The efficacy of silencing was verified by Western blot. d Silencing of both Stat1 and Stat3 expression prevented LPS-induced proinflammatory gene expression more efficiently than Jmjd3 silencing. Microglial cells were transfected with control siRNA or siRNAs specific for Stat1, Stat3, or Jmjd3. After $48 \mathrm{~h}$, the transfected cells were stimulated with LPS for $9 \mathrm{~h}$. The expression of selected genes in LPS-stimulated BV-2 cells was determined by quantitative PCR. For a specific experiment, measurements were compared to values for cultures transfected with control siRNA and stimulated with LPS. The graphs show means and standard deviations (s.d.) from three independent experiments. Statistical significance was calculated by comparison to cells transfected with control siRNA and stimulated with LPS. ${ }^{*} p<0.05$; ${ }^{*} p<0.01$; *** $p<0.001$ unaffected, as demonstrated by a representative immunoblot showing no changes in the phospho-IкB level (Fig. 4a). Forced expression of active Stat1 or Stat3 led to a significant but moderate increase in the expression of the selected inflammatory genes (Il-6, iNos, Ccl5, Pim1, Uba7, and Irf7) when compared to controls. Overexpression of Jmjd3 induced only a small increase in the expression of these selected genes. However, the expression of both Stat $1 \mathrm{C}$ and Stat $3 \mathrm{C}$ resulted in upregulation of the inflammatory gene expression levels to levels that were comparable or in some cases higher than those observed after LPS treatment (Fig. 4b). Furthermore, overexpression of Jmjd3 with constitutively active Stat3C or Stat1C yielded an upregulation of inflammatory gene expression to levels higher than those observed following LPS treatment.

To determine whether forced expression of Stat1C and/or Stat3C alone or in combination with Jmjd3 can induce inflammatory activation of microglial cells, we measured the secretion of pro-inflammatory cytokines in conditioned media using a multiplexed cytokine ELISA (Luminex). Surprisingly, 
overexpression of Stat1C and Stat3C individually or a combination of either of the constitutive Stats with Jmjd 3 increased the production of pro-inflammatory cytokines to levels similar to those observed following induction by LPS. The highest levels of Ccl5, IL-6, IFN- $\gamma$, IL-1 $\beta$, and TNF- $\alpha$ were detected in the conditioned media of cells overexpressing Stat1C and Stat3C or a combination of either of these Stats with Jmjd3. For IL10 (detected at low levels in all samples), forced expression of constitutively active Stat 1 and Stat3 resulted in a weak induction of cytokine production (Fig. 4c). Overexpression of Jmjd3 alone caused only a small increase in cytokine production.

Forced expression of constitutively active Stat1 and Stat3 mimics LPS-induced inflammatory responses in BV2 microglial cells

To assess whether the forced expression of Stat1C and Stat3C contributes to the global transcriptional network underlying inflammation, we performed gene expression profiling studies in BV2 microglial cells overexpressing Stat $1 \mathrm{C}$ and Stat3C or stimulated with LPS. Most of the genes whose expression levels were significantly altered following LPS treatment exhibited similar altered gene expression in Stat $1 \mathrm{C}+$ Stat3C-overexpressing cells. In most cases, overexpression of Stat1C and Stat3C resulted in larger changes in gene expression than those observed in cells induced with LPS (Fig. 5a).

We compared the percentage of genes whose expression levels changed in the same direction in the Stat $1 \mathrm{C}+\mathrm{Stat} 3 \mathrm{C}$ and LPS groups. Depending on the fold-change threshold used, the majority of genes $75-100 \%\left(p<10^{-12}\right)$ changed in the same direction in both groups (Fig. 5b). To determine whether changes in the expression levels in these groups correspond to the inflammatory signature, we used a GSEA [23]. In addition, inflammatory response classes from MSigDB gene collections were exploited to extract these genes from the LPS and Stat1C+Stat3C groups. For a majority of the classes, there was an enrichment of functional categories linked to the inflammatory response in both the LPS and Stat1C+Stat3C groups $(p<0.25$ significance threshold as suggested by GSEA authors), as shown in Fig. 5c. We also identified genes whose expression was significantly altered in opposite directions following LPS induction or Stat1C+Stat3C overexpression. The majority of these oppositely modulated genes $(95.8 \%)$ exhibited increased expression in the Stat $1 \mathrm{C}+\mathrm{Stat} 3 \mathrm{C}$ group and decreased expression in the LPS group. For these genes, we performed overrepresentation analysis using all of the classes from the MSigDB gene collection; nine of the classes appeared to be significantly altered (Fig. 5d). A majority of these classes are related to inflammatory and immune responses.
Jmjd3 acts independently of its demethylase activity in LPS-induced microglial cells

To determine whether histone H3K27me3 demethylation contributes to Jmjd3 activity in LPS-induced inflammatory gene expression in microglial cells, we used constructs encoding the following two mutants of Jmjd3: Jmjd3mut (deletion) [27] and Jmjd3mut (catalytic) [28]. The deletion mutant lacks the JmjdC domain, while the catalytic mutant contains three point mutations in the active site of the catalytic domain which affect H3K27me3 demethylase activity (Fig. 6a). The expression levels of the wild-type and the two mutant Jmjd3 constructs were similar (Fig. 6b). Forced expression of Jmjd3mut (catalytic) yielded the same changes in the expression levels of the selected genes as those observed following expression of wild-type Jmjd3. Deletion of the JmjdC domain abolished the Jmjd3-dependent transcription of $\mathrm{Il}-6$, iNos, and $\mathrm{Ccl} 5$ (Fig. 6b).

We further analyzed H3K27me3 demethylase activity to determine whether this activity contributes to global H3K27me3 levels in LPS-stimulated microglial cells. We determined the relative level of $\mathrm{H} 3 \mathrm{~K} 27 \mathrm{me} 3$ in LPSstimulated BV2 microglial cells by flow cytometry and demonstrated that neither Jmjd3 silencing nor Jmjd3 overexpression led to global changes in $\mathrm{H} 3 \mathrm{~K} 27 \mathrm{me} 3$ levels in LPS-stimulated microglial cells (Fig. 6c). As a positive control, we used microglial cells pretreated with epigenetic enzyme inhibitors (Fig. 6d). Taken together, these results demonstrate that the H3K27me3 activity of Jmjd3 does not contribute to its role in inducing the expression of inflammatory genes.

Stat 1 and Stat 3 activation is a secondary response driven by early cytokine release

To elucidate upstream events leading to LPS-induced Stat activation, we determined the levels of phosphorylated Stats in primary microglial cultures from various Tlr knockout mice. We found that LPS does not induce Stat phosphorylation (Fig. 7a) and upregulation of inflammatory gene expression (Fig. 7b) in microglia isolated from Tlr4 knockout mice. Both Stat phosphorylation and upregulation of inflammatory genes were not affected in microglial cultures from Tlr1, 2, 7, and 9 knockouts (not shown). Addition of a medium from LPS-stimulated microglia from Tlr4wt mice to Tlr4KO microglial cultures restored Stat phosphorylation and gene upregulation (Fig. 7b). It suggests that LPS via Tlr4 induces secreted factors, which in turn activate Stats. This notion is supported by the results of the experiment with LPS stimulation in the presence of CHX, a protein synthesis inhibitor. CHX blocks Stat phosphorylation (Fig. 7c) and significantly reduces the expression of inflammatory genes (Il-6, iNos, Ccl5, and Pim 1) after the LPS treatment (Fig. 7d). In 


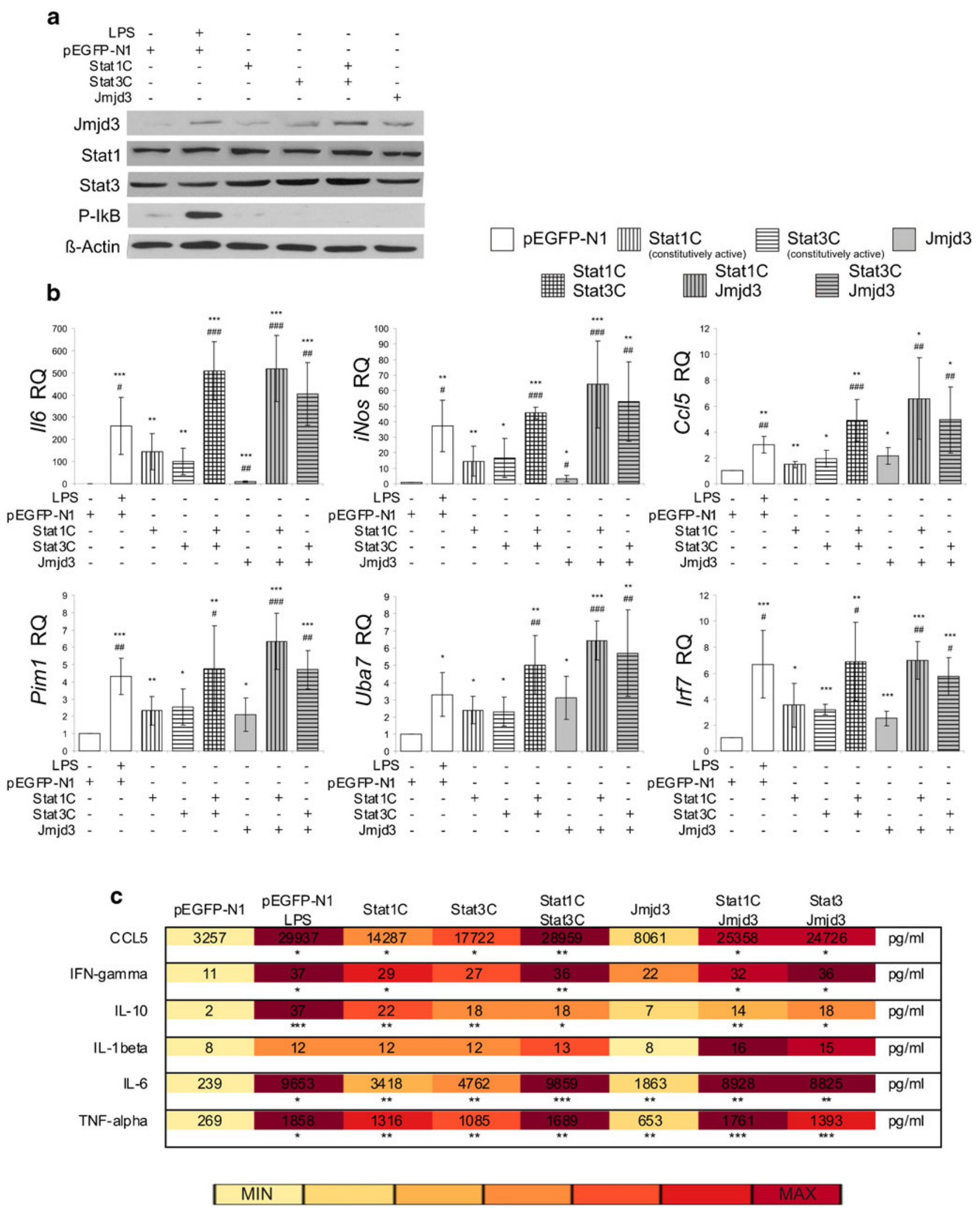

microglial cells Stat1 phosphorylation was induced by a recombinant IFN- $\gamma$ and Stat3 phosphorylation by IL-6, respectively (Fig. 7e). The increased expression of these cytokines (as well as Ifn- $\beta$ and $T n f-\alpha$ ) at the mRNA level was 
Fig. 4 Overexpression of active Stat1 and Stat3 alone or in combination with Jmjd3 induces the transcription of inflammatory genes and cytokine secretion. a Evaluation of the expression levels of Stat1, Stat3, Jmjd3, and PI $\mathrm{B}$ proteins in microglial cells overexpressing specific proteins. BV2 microglial cells were transfected by an Amaxa protocol with constructs encoding constitutively active Stat1, Stat3, Jmjd3, or a pEGFP-N1 control. Twenty-four hours post-transfection, the cells were left untreated or were stimulated with LPS for $9 \mathrm{~h}$. b Relative quantification (qPCR) of selected gene expression in BV2 cells transfected with constructs encoding constitutively active Stat1, Stat3, Jmjd3, pEGFP-N1, or stimulated with LPS. Graphs show mean \pm SD for four independent experiments. In a given experiment, measurements were compared to the values obtained for pEGFP-N1transfected cells (statistical significance was calculated for comparisons of cells transfected with pEGFP-N1 versus other cells: * $p<0.05$; ${ }^{*} p<0.01$; $*_{* *} p<0.001$; cells transfected with a single construct of Stat 1 or Stat 3 versus other cells: $\left.{ }^{\#} p<0.05 ;{ }^{\#} p<0.01 ;{ }^{\# \#} p<0.001\right)$. c Heatmap representation of the selected cytokine concentrations in conditioned media collected from BV2 microglial cells transfected with constructs encoding constitutively active Stat1, Stat3, Jmjd3, pEGFP-N1, or stimulated with LPS (from the same experiments as described above). Conditioned media were collected $24 \mathrm{~h}$ after transfections or $9 \mathrm{~h}$ after LPS stimulation and processed for multiplex cytokine ELISAs using the Luminex system. A color-coded representation of cytokine concentrations is presented. The heatmap shows means of three independent experiments; statistical significance was calculated by comparing cells transfected with pEGFP-N1 with other cells: ${ }^{*} p<0.05$; ${ }^{* *} p<0.01 ;{ }^{* * *} p<0.001$ detected as soon as 15 min after LPS stimulation. The second increase in their mRNA levels was observed 1 and $1.5 \mathrm{~h}$ after the LPS treatment (Fig. 7f).

\section{Discussion}

The main findings of this study are summarized as follows: (1) Stat 1 and Stat 3 binding peaks were associated with the genes most strongly induced by LPS in microglial cells; (2) chromatin immunoprecipitation revealed that Jmjd3 is a direct target of Stat1 and Stat3; (3) silencing of both Stat1 and Stat3 strongly affected novel and known Stat target genes in LPS-stimulated microglial cells; (4) the forced expression of constitutively active Stat1 and Stat3 was sufficient to induce the transcription of hundreds of genes, mimicked LPS stimulation and resulted in the production of inflammatory cytokines; (5) Jmjd3 alone weakly induced gene expression, but Jmjd3 in cooperation with either Stat induced a complete inflammatory response; (6) the transcriptional effects of Jmjd3 overexpression appeared to be independent of its H3K27me3 demethylase activity; and (7) phosphorylation of

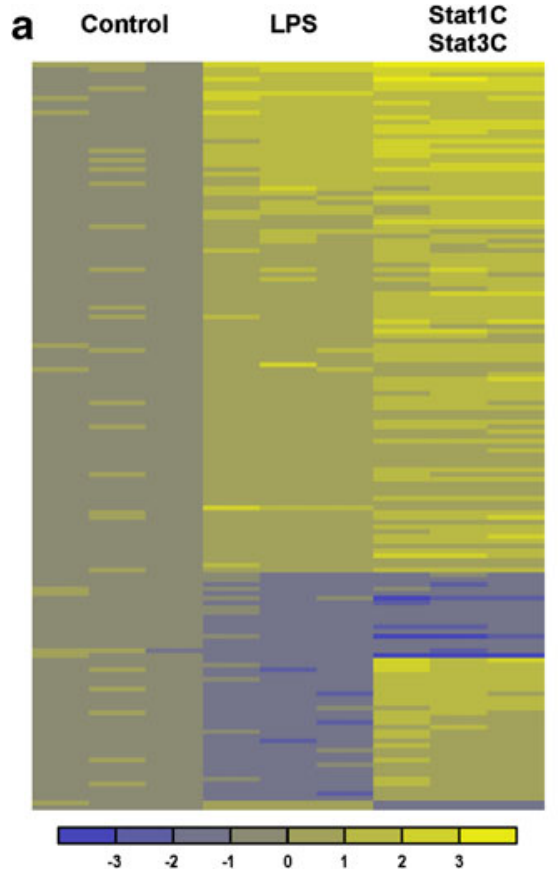

Fig. 5 Overexpression of constitutive Stat $1 \mathrm{C}$ and Stat $3 \mathrm{C}$ mimics transcriptional changes induced by LPS in BV2 microglial cells. a Global gene expression was studied in untreated pEGFP-N1-transfected BV2 microglial cells, cells overexpressing constitutively active Stat1C and Stat $3 \mathrm{C}$ or cells treated for $9 \mathrm{~h}$ with LPS, $n=3 /$ group. Heatmap representation of RMA-normalized microarray expression data for genes whose expression was significantly altered in LPS-treated cells or cells overexpressing constitutively active Stat $1 \mathrm{C}$ and Stat3C compared to cells transfected with pEGFP-N1 $(q<0.05$ and FC $>1.5$ or $<0.66)$. For a given gene, an average value in the control samples was computed and subtracted from each value in an experimental sample. For the heatmap

b

d

\begin{tabular}{|c|c|c|}
\hline Fold Crange & No. of genes (all=427 4 ) & $\begin{array}{c}\text { Geres charged in } \\
\text { the same eirection }\end{array}$ \\
\hline 1,2 & 873 & $75 \%$ \\
1,5 & 132 & $78 \%$ \\
1,7 & 56 & $85 \%$ \\
2 & 26 & $92 \% \%$ \\
3 & 7 & $100 \%$ \\
\hline
\end{tabular}

C

\begin{tabular}{|c|c|c|}
\hline Inflamatory response classes from MSig DB gene collections & LPS & $\begin{array}{l}\text { Sta1C } \\
\text { Sta3C }\end{array}$ \\
\hline FOSTER_INFLAMMATORY_RESPONSE_LPS_UP & $p<0.0001$ & $p<0.0001$ \\
\hline FOSTER_INFLAMMATORY_RESPONSE_LPSDDN & $p<0.0001$ & $p<0.0001$ \\
\hline SEW_INFLAMMATORY_FESPONSE_IPS_UP & $p<0.0001$ & $p<0.0001$ \\
\hline SEEI_INFLAMMATORY_RESPONSE_LPS_DN & $\mathrm{p}=0.8877$ & $\mathrm{p}=0.0004$ \\
\hline COATES_MACROPHACE_M1_VS_MR_UP & $\mathrm{p}=0.0713$ & $\mathrm{p}=0.0937$ \\
\hline COATES MACROPHAGE M1 VS M2 DN & $0=02472$ & $0=03772$ \\
\hline
\end{tabular}

\begin{tabular}{|c|c|}
\hline Classes from MSigDB gene collections & p-value \\
\hline 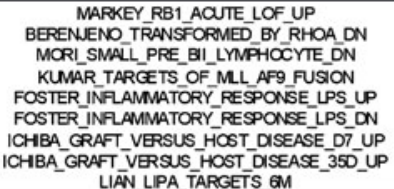 & $\begin{array}{l}p<0.0001 \\
p=0.0294 \\
p=0.0473 \\
p=0.0156 \\
p<0.0001 \\
p<0.0001 \\
p<0.0001 \\
p=0.0002 \\
p=0.0174\end{array}$ \\
\hline
\end{tabular}

presentation, values higher than 3 were set to 3 . b Influence of foldchange threshold on the number of genes whose expression was altered and on the percentage of genes modulated in the same direction in microglial cells treated with LPS and cells overexpressing constitutively active Stat1C and Stat3C. $\mathbf{c}$ Gene set enrichment analysis (GSEA) of the LPS and Stat $1 \mathrm{C}+$ Stat $3 \mathrm{C}$ data sets using inflammatory response classes from the MSigDB gene collections. d Overrepresentation analysis using classes from the MSigDB gene collections on genes whose expression levels were significantly altered in opposing directions in LPS and Stat $1 \mathrm{C}+$ Stat3C data sets 
a

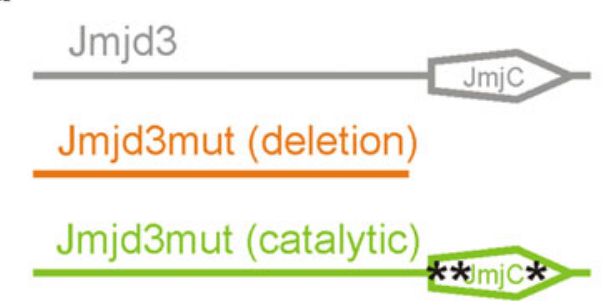

\section{b}

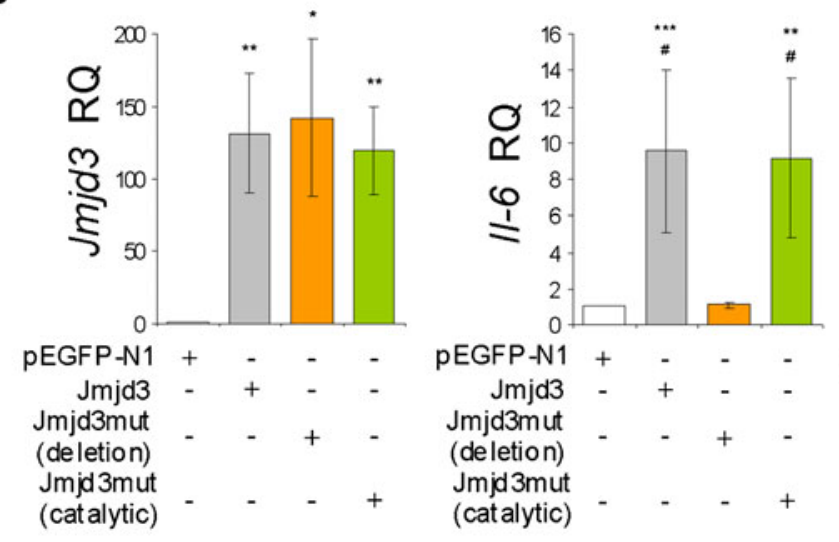

Fig. 6 H3K27me3 demethylase activity of Jmjd3 is not required for the regulation of transcriptional responses in microglial cells. a A graphic depicts the Jmjd3 constructs used in the experiments. Cells were transfected with constructs encoding wild-type Jmjd3, a deletion mutant of Jmjd3 (lacking the JmjdC catalytic domain), and a catalytic mutant of Jmjd3 (containing three point mutations in the catalytic center that completely abrogate demethylase activity). b Quantification of the expression of Jmjd 3 and three selected inflammatory genes (Il-6, iNos, and Ccl5) in microglial cells transfected with pEGFP-N1 or Jmjd3 constructs. Total RNA was isolated $24 \mathrm{~h}$ after transfection using Cell-to-CT assays,

Stat 1 and Stat3 is induced by an early cytokine release triggered by LPS-induced stimulation of Tlr4 on microglial cells.

Based on this work, we propose a model for the molecular events triggered by LPS treatment in which microglial cells launch the inflammatory response (Fig. 8). First, LPS binds to TLR4 receptor and stimulates early cytokine expression (via NF-KB or mRNA stabilization). These cytokines when released induce Stat1 and Stat3 activation. Stat dimers in combination with NF-KB upregulate Jmjd3 expression. Next, Stat1 and Stat3, in concert with Jmjd3, activate a transcriptional network that results in functional, inflammatory activation of microglia.

A recent meta-analysis of $29 \mathrm{ChIP}$-seq data sets covering genome-wide occupancy of STATs in several cell types, mostly under basal conditions [16] revealed that each cell type displays a unique STAT binding pattern, for that reason transcriptional Stat targets could vary in distinct cells. Therefore, to understand functions of specific Stats, it is crucial to

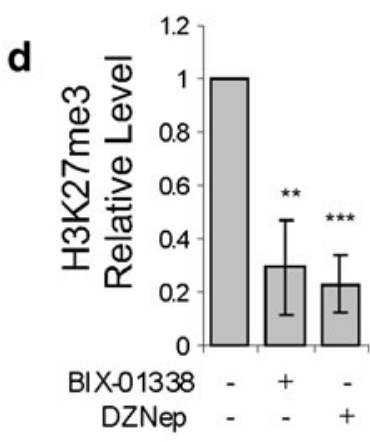

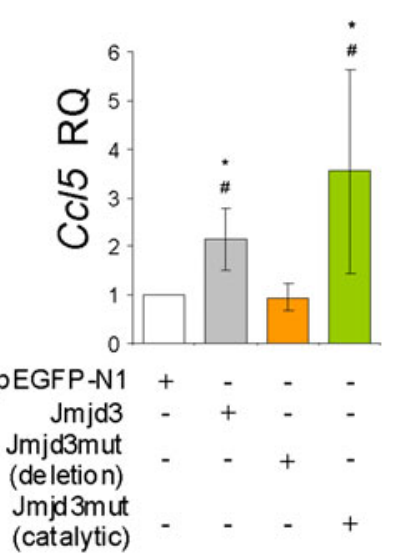

and gene expression was determined by qPCR. Graphs show means \pm SD of three independent experiments (statistical significance for Jmjd3 constructs versus the pEGFP-N1-transfected control cells ${ }^{*} p<0.05 ; *^{*} p<$ $0.01 ; * * * p<0.001$; or Jmjd3mut(deletion) transfected cells versus other groups: $\left.{ }^{\#} p<0.05 ;{ }^{\# \#} p<0.01 ;{ }^{\# \#} p<0.001\right)$. c Overexpression of Jmjd3 deficient in H3K27me3 demethylase activity does not modulate global changes in H3K27me3 levels as determined by flow cytometry. d In the control experiment global H3K27me3 levels were evaluated in the cells treated for $6 \mathrm{~h}$ with histone methyltransferase inhibitors: $3 \mu \mathrm{M} \mathrm{3-}$ deazaneplanocin or $3 \mu \mathrm{M}$ BIX 01294

establish Stat genome-wide occupancy in specific cells and under given conditions. A microglial cell is both a glial cell of the central nervous system (CNS) and a mononuclear phagocyte. Microglial progenitors colonize the CNS primarily during embryonic and fetal periods of development. In the adult CNS, they act as sentinels of infection and injury, and participate in both innate and adaptive immune responses [29]. We demonstrated for the first time that Stat1, Stat3, and Stat5 binding sites are widely distributed in the microglial genome. Although many Stat1 and Stat3 binding sites were positively correlated with genes that are highly upregulated in the LPSstimulated microglial cells, we also identified many binding sites in the promoter regions of genes whose expression was unaltered in response to treatment. This suggests that the binding of active Stat1 or Stat3 alone is not sufficient to modulate the expression of these genes. Most of the tested genes contained binding peaks for either Stat1 or Stat3. Given the high similarity of the binding sites for Stat1 and Stat3, 

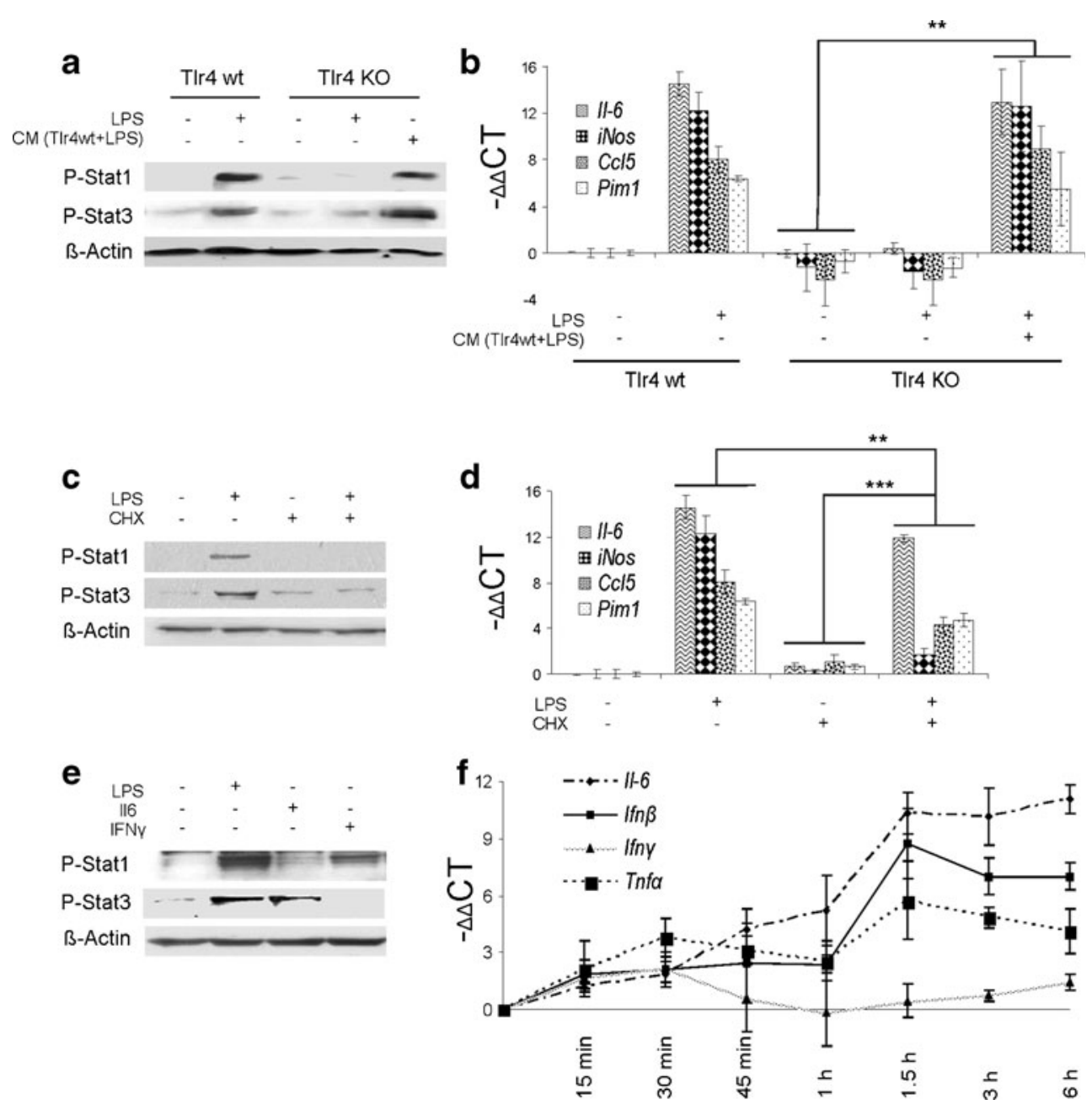

Fig. 7 In LPS-stimulated microglia Stat1 and Stat3 phosphorylation is driven by Tlr4 activation and early cytokine release. a The levels of phosphorylated Stat1 and Stat3 in total protein extracts from primary microglial cultures isolated from Tlr4 wild-type and knockout mice, unstimulated or stimulated for $3 \mathrm{~h}$ with LPS. Addition of a conditioned medium from LPS-stimulated microglia from Tlr4wt mice to Tlr4KO microglial cultures restored Stat phosphorylation and gene upregulation. b Relative quantification (qPCR) of selected gene expression in primary microglia cultures isolated from Tlr4 wild-type and knockout mice, unstimulated or stimulated by LPS for $3 \mathrm{~h}$. A conditioned medium from LPS-stimulated microglia from Tlr4wt mice restored Stat phosphorylation and gene upregulation in Tlr4KO microglial cultures. The results are shown as $-\Delta \Delta \mathrm{CT}\left(\log _{2} R Q\right)$. Statistical significance: ${ }^{*} p<0.05 ;{ }^{* *} p<$
$0.01 ; * * * p<0.001$. c The levels of phosphorylated Stat1 and Stat 3 in total protein extracts from primary microglial cultures unstimulated or stimulated for $3 \mathrm{~h}$ with LPS in the absence or presence of cycloheximide. d Quantification (qPCR) of selected gene expression in primary microglia cultures unstimulated or stimulated for $6 \mathrm{~h}$ with LPS in the absence or presence of cycloheximide. The results are shown as $-\Delta \Delta \mathrm{CT}\left(\log _{2} R Q\right)$. Statistical significance: ${ }^{*} p<0.05$; ${ }^{*} p<0.01 ;{ }^{* *} p<0.001$. e The levels of phosphorylated Stat1 and Stat3 in total protein extracts from murine primary microglial cultures untreated or exposed for $3 \mathrm{~h}$ with LPS or recombinant murine cytokines Il-6 or Ifn- $\gamma$. f Relative quantification (qPCR) of the levels of Ifn- $\gamma$, Il- 6 , Ifn- $\beta$, and Tnf- $\alpha$ mRNAs in primary microglial cultures at various times after the treatment with LPS these results suggest that Stat 1 and Stat 3 can each compensate for the function of the other Stat protein.

We identified a subset of Stat binding genes that were highly upregulated in inflammatory microglial cells (Fig. 2) including $I l-$ 6, a known target of Stats, and several novel targets: Jmjd3, Ccl5, Ezr, Ifih1, Irf7, Uba7, and Pim1. These results demonstrate that Stat1 and Stat3 are required to activate the expression of genes coding for proteins important in the inflammatory response. C-C motif ligand 5 (Ccl5/RANTES - regulated upon activation, normal $\mathrm{T}$ cell expressed and secreted) is a chemokine that facilitates the induction of chemotaxis in immune cells under conditions of atherosclerosis and cerebral infarction [30]. The Ifih1 gene encodes IFIH1 (interferon induced with helicase C domain 1), an interferon-induced helicase known to mediate an innate immune response [31]. Uba7 (UbelL) encodes the key enzyme that activates ISGylation, an interferon-inducible, ubiquitination-like post-translational protein modification system [32]. IRF 7 is a transcription factor that mediates interferon- $\gamma$ and Toll-like receptor signaling. IRF7 expression and activity is indispensable for appropriate type I IFN production and IFN- 
Fig. 8 Model depicting the role of the Stat proteins in the regulation of inflammatory transcriptional responses in microglia. LPS activates TLR4 receptor that results in NF-kB activation and early cytokine production. Released cytokines leads to phosphorylation of Stat1 and Stat3. Activation of NF- $k B$, Stat1, and Stat3 leads to the expression of Jmjd3, which, together with Stat1 and Stat3, drives the expression of proinflammatory genes. The dashed line represents relations which have not been studied in this work

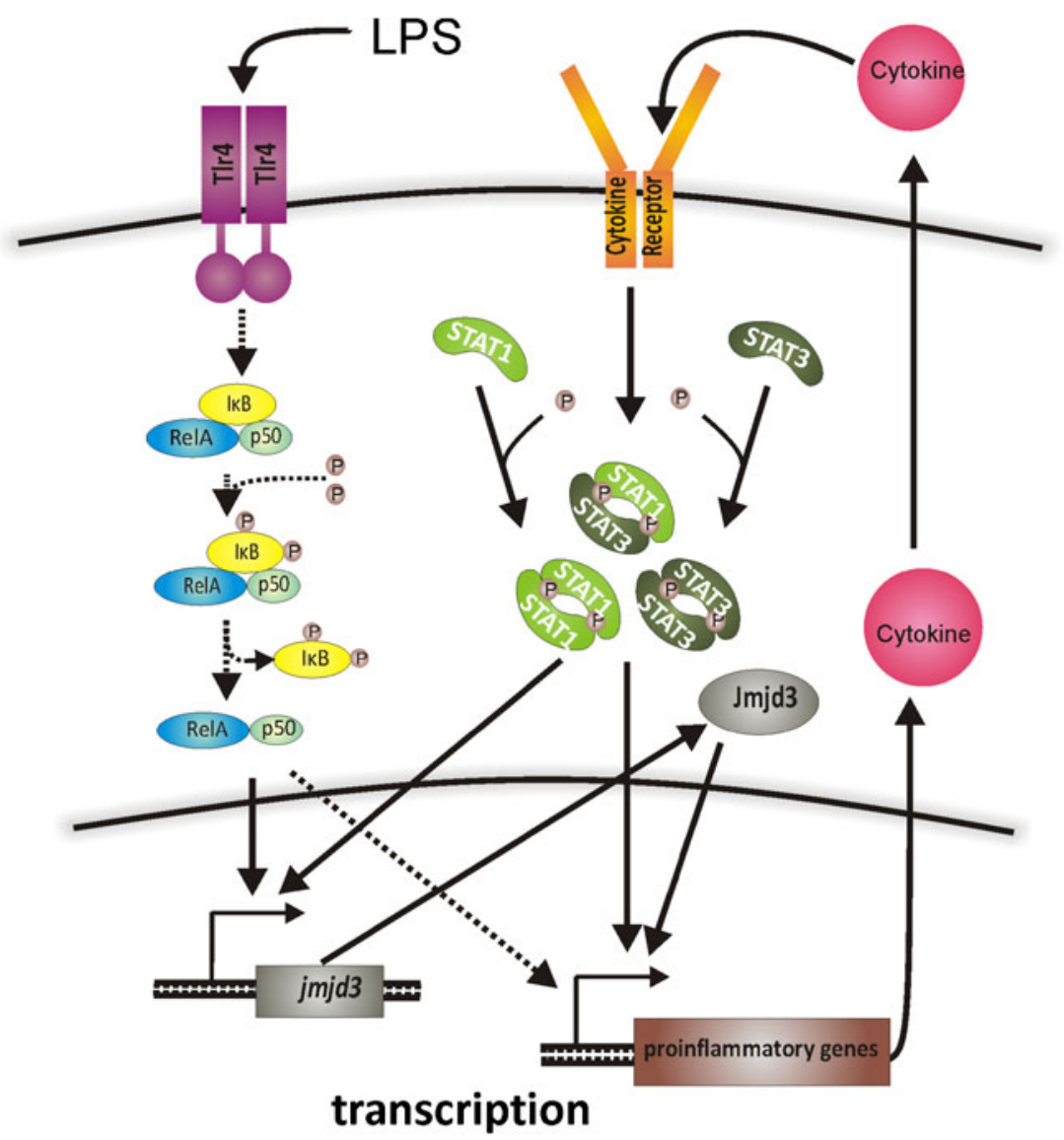

mediated physiological functions [33]. Pim1 belongs to a family of serine/threonine protein kinases that are involved in the control of cell growth, differentiation and apoptosis. Pim1 can be induced by several external stimuli, including a number of cytokines relevant to the immune response [34]. Our results provide new evidence for direct interactions between Stats and the Pim1, Jmjd3, Ccl5, Ezr, Ifih1, Irf7, and Uba7 gene promoters.

We focused on the role of Stats in the regulation of the Jmjd3 gene, which we identified as a new transcriptional Stat target. The Jmjd3 gene has both Stat1 and Stat 3 binding sites within its promoter region. Jmjd 3 is a JmjC family member and a histone demethylase implicated in $\mathrm{H} 3 \mathrm{~K} 27 \mathrm{me} 3$ demethylation, which erases the transcriptional repression involved in lineage determination. Jmjd3 is an important regulator of inflammatory gene transcription in peripheral macrophages $[25,35]$. The relevance of the demethylase activity of JMJ enzymes in regulating cellular responses is unclear. De Santa and colleagues [25] reported that Jmjd3, induced by LPS and interferon- $\gamma$ stimulation, associates with inflammation-related genes in peripheral macrophages. However, most Jmjd3 target genes were not associated with detectable levels of $\mathrm{H} 3 \mathrm{~K} 27 \mathrm{me} 3$, and the expression of Jmjd3 binding genes was unaffected or only moderately impaired by a mutant Jmjd3 with impaired demethylase activity. It suggests that Jmjd3 regulates the transcriptional response in a H3K27 demethylation-independent manner [25]. However, a recent study showed that a small-molecule inhibitor of the H3K27me3-specific JMJ subfamily reduces LPS-induced inflammatory cytokine production by human primary macrophages [25]. We found that inhibition of the demethylase activity of Jmjd3 did not alter the expression of the inflammatory Jmjd3-dependent genes and had no effect on global H3K27me3 levels in LPS-activated microglial cells (Fig. 5) that support a notion that Jmjd3 regulates the transcriptional, inflammatory response in a H3K27 demethylation-independent manner.

This is a first report that provides evidence for Statdependent regulation of Jmjd3 expression during LPSinduced inflammatory responses. (Fig. 3b); however, NF-kB also participates in the transcriptional regulation of Jmjd3. The effects of Stat 1 and Stat 3 silencing or the ectopic expression of persistently activated Stat 1 and Stat 3 on inflammation-related genes are much stronger than the changes induced by Jmjd3 silencing or overexpression. This suggests that Stat1 and Stat3 contribute to the induction of gene expression not only through Jmjd3 activation but also by regulating transcriptional changes themselves (Fig. 3). Forced Jmjd3 expression in microglial cells resulted in minor effects on the mRNA levels of inflammatory genes and a modest effect on inflammatory cytokine production (Fig. 4) compared to LPS or Stat overexpression. However, Jmjd3 cooperated with the constitutively active Stat1C and Stat3C to upregulate the mRNA levels of 
inflammatory genes and cytokine production to levels comparable to those observed following LPS stimulation (Fig. 4). These results suggest that Jmjd3 cooperates with Stat1 and Stat 3 to additionally alter the expression level. Although data regarding Jmjd3 DNA-binding sites in LPS-stimulated rat microglial cells are not available, computational comparison of our Stat binding data and Jmjd3 binding data from LPSstimulated mouse intraperitoneal macrophages [25] reveals a significant correlation $\left(p<10^{-12}\right)$. This suggests that similar target genes are regulated by Stat1, Stat3, and Jmjd3, which cooperate to fully activate transcriptional responses during the inflammatory response.

Interestingly, forced expression of Stat1C and Stat3C leads to cytokine production (Fig. 4c); therefore, other signaling events are initiated in addition to the activation of transcriptional machinery. Stat1C and Stat3C overexpression was more effective and produced larger changes than LPS treatment, with the exception of IL10 production. IL10 is an anti-inflammatory cytokine and participates in the resolution of the inflammatory response. Therefore, while activation of Stat1 and Stat3 seems to be sufficient to activate inflammation, it cannot support a complex physiological response that involves termination of inflammation.

The analysis of upstream signaling events leading to Stat activation shows that Tlr4 knockout impaired LPS-induced Stat phosphorylation and pro-inflammatory gene expression in primary microglial cultures. However, the effect of Tlr4 signaling on Stat activation in microglial cells is indirect and likely caused by early cytokine release (Fig. 7). Similar mechanism was shown earlier in a case of TLR4-NF-KB-IL-6-Stat3 signaling in an experimental model of LPS/TLR4-mediated septic shock [13]. It suggests that a loop consisting of early released cytokines (IFN- $\gamma$ and IL-6) and activated Stats is responsible for most of changes occurring in inflammatory microglia.

Altogether, our results demonstrate that Stat1 and Stat3 are essential, and sufficient for initiating an appropriate inflammatory response. This challenges the emerging idea that blocking Stat3 may inhibit antitumor immune responses. While Stat 1 has been repeatedly linked to the initiation of classical inflammatory responses, Stat 3 is thought to act as an oncogene in cancer cells, and as an inducer of immunosuppression when activated in immune cells within the tumor microenvironment. There have been many attempts to block Stat3 expression or activity in immune cells located within the tumor microenvironment [6]. Our results show that both Stat 1 and Stat 3 contribute to activating the expression of inflammatory genes. It raises concerns that blocking Stat3 may not be a good strategy for restoring the expression of the mediator proteins necessary to activate the immune response against tumor cells.

Acknowledgments We would like to thank Dr. Marta Maleszewska for assistance in determining the H3K27me3 levels by flow cytometry (experiments were performed in the Laboratory of Cytometry of the Nencki Institute of Experimental Biology) and Anna Siuda for assistance in determining cytokine levels with the Luminex system. We would like to thank Prof. Dr. Seija Lehnardt, Karen Rosenberger, and Dr. Katja Derkow from Charite Medical University, Berlin (who organized breeding and transport of all TlrKO mice). We would like to thank ImmunoTools for providing us with recombinant cytokines (IT-Box-Cy55M ImmunoTools Award 2013). Studies were supported by grants (N301 239536) from the Ministry of Science and Higher Education (JK) and (2011/03/N/NZ1/ 03143) from the Polish National Science Centre (PP), and the Foundation for Polish Science, the International PhD Projects program (PP). The funders had no role in the study design, data collection, and analysis, decision to publish, or preparation of the manuscript.

Conflict of interest All authors declare no conflict of interest.

Open Access This article is distributed under the terms of the Creative Commons Attribution License which permits any use, distribution, and reproduction in any medium, provided the original author(s) and the source are credited.

\section{References}

1. Nicodeme E, Jeffrey KL, Schaefer U, Beinke S, Dewell S, Chung CW, Chandwani R, Marazzi I, Wilson P, Coste H et al (2010) Suppression of inflammation by a synthetic histone mimic. Nature 468:1119-1123

2. O'Shea JJ, Plenge R (2012) JAK and STAT signaling molecules in immunoregulation and immune-mediated disease. Immunity 36:542550

3. Ivashkiv LB, Hu X (2004) Signaling by STATs. Arthritis Res Ther 6: $159-168$

4. Hayden MS, West AP, Ghosh S (2006) NF-kappaB and the immune response. Oncogene 25:6758-6780

5. Honda K, Taniguchi T (2006) IRFs: master regulators of signalling by Toll-like receptors and cytosolic pattern-recognition receptors. Nat Rev Immunol 6:644-658

6. Yu H, Kortylewski M, Pardoll D (2007) Crosstalk between cancer and immune cells: role of STAT3 in the tumour microenvironment. Nat Rev Immunol 7:41-51

7. Kaminska B, Swiatek-Machado K (2008) Targeting signaling pathways with small molecules to treat autoimmune disorders. Expert Rev Clin Immunol 4:93-112

8. Akira S (1999) Functional roles of STAT family proteins: lessons from knockout mice. Stem Cells 17:138-146

9. Meraz MA, White JM, Sheehan KC, Bach EA, Rodig SJ, Dighe AS, Kaplan DH, Riley JK, Greenlund AC, Campbell D et al (1996) Targeted disruption of the Statl gene in mice reveals unexpected physiologic specificity in the JAK-STAT signaling pathway. Cell 84: 431-442

10. Bromberg JF, Wrzeszczynska MH, Devgan G, Zhao Y, Pestell RG, Albanese C, Darnell JE (1999) Stat3 as an oncogene. Cell 98:295-303

11. Takeda K, Clausen BE, Kaisho T, Tsujimura T, Terada N, Förster I, Akira S (1999) Enhanced Th1 activity and development of chronic enterocolitis in mice devoid of Stat3 in macrophages and neutrophils. Immunity 10:39-49

12. Kano A, Wolfgang MJ, Gao Q, Jacoby J, Chai GX, Hansen W, Iwamoto Y, Pober JS, Flavell RA, Fu XY (2003) Endothelial cells require STAT3 for protection against endotoxin-induced inflammation. J Exp Med 198:1517-1525

13. Greenhill CJ, Rose-John S, Lissilaa R, Ferlin W, Ernst M, Hertzog PJ, Mansell A, Jenkins BJ (2011) IL-6 trans-signaling modulates TLR4dependent inflammatory responses via STAT3. J Immunol 186: 1199-1208 
14. Sikorski K, Czerwoniec A, Bujnicki JM, Wesoly J, Bluyssen HA (2011) STAT1 as a novel therapeutical target in pro-atherogenic signal integration of IFN $\gamma$, TLR4 and IL-6 in vascular disease. Cytokine Growth Factor Rev 22:211-219

15. Kornfeld JW, Grebien F, Kerenyi MA, Friedbichler K, Kovacic B, Zankl B, Hoelbl A, Nivarti H, Beug H, Sexl V et al (2008) The different functions of Stat5 and chromatin alteration through Stat5 proteins. Front Biosci 13:6237-6254

16. Kang K, Robinson GW, Hennighausen L (2013) Comprehensive metaanalysis of signal transducers and activators of transcription (STAT) genomic binding patterns discerns cell-specific cis-regulatory modules. BMC Genomics 14:4

17. Glass CK, Saijo K, Winner B, Marchetto MC, Gage FH (2010) Mechanisms underlying inflammation in neurodegeneration. Cell 140:918-934

18. Zawadzka M, Dabrowski M, Gozdz A, Szadujkis B, Sliwa M, Lipko M, Kaminska B (2012) Early steps of microglial activation are directly affected by neuroprotectant FK506 in both in vitro inflammation and in rat model of stroke. J Mol Med (Berl) 90:1459-1471

19. Hoshino K, Takeuchi O, Kawai T, Sanjo H, Ogawa T, Takeda Y, Takeda K, Akira S (1999) Cutting edge: Toll-like receptor 4 (TLR4)deficient mice are hyporesponsive to lipopolysaccharide: evidence for TLR4 as the Lps gene product. J Immunol 162:3749-3752

20. Sliwa M, Markovic D, Gabrusiewicz K, Synowitz M, Glass R, Zawadzka M, Wesolowska A, Kettenmann H, Kaminska B (2007) The invasion promoting effect of microglia on glioblastoma cells is inhibited by cyclosporin A. Brain 130:476-489

21. Mieczkowski J, Tyburczy ME, Dabrowski M, Pokarowski P (2010) Probe set filtering increases correlation between Affymetrix GeneChip and qRT-PCR expression measurements. BMC Bioinforma 11:104

22. Gentleman RC, Carey VJ, Bates DM, Bolstad B, Dettling M, Dudoit S, Ellis B, Gautier L, Ge Y, Gentry J et al (2004) Bioconductor: open software development for computational biology and bioinformatics. Genome Biol 5:R80

23. Subramanian A, Tamayo P, Mootha VK, Mukherjee S, Ebert BL, Gillette MA, Paulovich A, Pomeroy SL, Golub TR, Lander ES et al (2005) Gene set enrichment analysis: a knowledge-based approach for interpreting genome-wide expression profiles. Proc Natl Acad Sci U S A 102:15545-15550
24. De Santa F, Totaro MG, Prosperini E, Notarbartolo S, Testa G, Natoli G (2007) The histone H3 lysine-27 demethylase Jmjd3 links inflammation to inhibition of polycomb-mediated gene silencing. Cell 130: 1083-1094

25. De Santa F, Narang V, Yap ZH, Tusi BK, Burgold T, Austenaa L, Bucci G, Caganova M, Notarbartolo S, Casola S et al (2009) Jmjd3 contributes to the control of gene expression in LPS-activated macrophages. EMBO J 28:3341-3352

26. Sironi JJ, Ouchi T (2004) STAT1-induced apoptosis is mediated by caspases 2, 3, and 7. J Biol Chem 279:4066-4074

27. Solá S, Xavier JM, Santos DM, Aranha MM, Morgado AL, Jepsen $\mathrm{K}$, Rodrigues CM (2011) p53 interaction with JMJD3 results in its nuclear distribution during mouse neural stem cell differentiation. PLoS One 6:e18421

28. Miller SA, Mohn SE, Weinmann AS (2010) Jmjd3 and UTX play a demethylase-independent role in chromatin remodeling to regulate Tbox family member-dependent gene expression. Mol Cell 40:594 605

29. Saijo K, Glass CK (2011) Microglial cell origin and phenotypes in health and disease. Nat Rev Immunol 11:775-787

30. Mirabelli-Badenier M, Braunersreuther V, Viviani GL, Dallegri F, Quercioli A, Veneselli E, Mach F, Montecucco F (2011) CC and CXC chemokines are pivotal mediators of cerebral injury in ischaemic stroke. Thromb Haemost 105:409-420

31. Yoneyama M, Kikuchi M, Matsumoto K, Imaizumi T, Miyagishi M, Taira K, Foy E, Loo YM, Gale M, Akira S et al (2005) Shared and unique functions of the DExD/H-box helicases RIG-I, MDA5, and LGP2 in antiviral innate immunity. J Immunol 175: 2851-2858

32. Dao CT, Zhang DE (2005) ISG15: a ubiquitin-like enigma. Front Biosci 10:2701-2722

33. Ning S, Pagano JS, Barber GN (2011) IRF7: activation, regulation, modification and function. Genes Immun 12:399-414

34. Bachmann M, Möröy T (2005) The serine/threonine kinase Pim-1. Int J Biochem Cell Biol 37:726-730

35. Kruidenier L, Chung CW, Cheng Z, Liddle J, Che K, Joberty G, Bantscheff M, Bountra C, Bridges A, Diallo H et al (2012) A selective jumonji H3K27 demethylase inhibitor modulates the proinflammatory macrophage response. Nature 488:404-408 